\title{
Eine Analyse ultimaten Verhaltens als Erklärungsansatz des moral hazards
}

\author{
Gottfried Koch $\cdot$ Julia Ostner $\cdot$ Marco Peisker $\cdot$ Oliver Schülke
}

Zusammenfassung Der moral hazard stellt für das Versicherungsunternehmen ein schwer kalkulierbares Risiko dar, weil Verhaltensänderungen des Versicherungsnehmers zu höheren Schadenzahlungen für den Versicherer und dadurch zu höheren Prämien für das gesamte Kollektiv führen können. Dem versicherten Individuum bietet die Informationsasymmetrie die Möglichkeit, den Schutzgedanken des Prinzips Versicherung zu seinem persönlichen Vorteil zu untergraben und somit den Versicherer und das gesamte Kollektiv zu schädigen. Ökonomische Ansätze haben, aufbauend auf dem Modell des homo oeconomicus ', versucht, Lösungsansätze für diese Gefahr aufzuzeigen. Verhaltensökonomische Experimente haben hingegen demonstriert, dass das Modell des homo oeconomicus nicht ausreichend genug in der Lage ist, die Realität zu beschreiben, weswegen die Lösungsvorschläge der ökonomischen Ansätze als unzureichend zu bezeichnen sind.

Moral hazard steht im Spannungsfeld von Kooperation und Defektion, Verhaltensweisen, die sich im Laufe der menschlichen Entwicklungsgeschichte evolviert haben. Ultimate Verhaltensanalysen dieser Verhaltensweisen bieten die Chance, die Frage nach der Grundursache aus deren evolutionären Ursprüngen und Quellen heraus zu verstehen. Deswegen dürften ultimate Verhaltensanalysen auch eine geeignetere Grundlage zur Entwicklung eines Rahmenwerkes bilden, das es Versicherern

\footnotetext{
G. Koch ( $)$ M. Peisker

Universität Leipzig,

Leipzig, Deutschland

E-Mail: koch@informatik.uni-leipzig.de

J. Ostner · O. Schülke

Georg August Universität,

Göttingen, Deutschland

J. Ostner · O. Schülke

Max Planck Institut für evolutionäre Anthropologie,

Leipzig, Deutschland
} 
ermöglichen könnte, das individuelle Verhalten des Versicherten so zu beeinflussen, dass dieser auf eine für Versicherungsunternehmen und Kollektiv negative Verhaltensänderung nach Abschluss des Versicherungsvertrages tendenziell verzichtet und die Gefahr des moral hazard so reduziert wird.

Abstract For the insurance industry the moral hazard poses an incalculable risk. Changes in the behaviour of the insuree can lead to higher claims settlements and thus to higher premiums for the insured collective. Asymmetrically allocated information gives the insured individual the possibility to profit from the idea of common protection in a way that impairs insurer and the collective on the whole. Based on the homo oeconomicus model, economic concepts, first and foremost the agency theory of new institutional economics have made attempts to provide solutions for this management problem. However, behavioural economic experiments have demonstrated that the homo oeconomicus model does not fully succeed in describing the realities. As a result, the solutions proposed in these economic concepts have to be rated inadequate.

The moral hazard is inherent in the conflicting realm of cooperation and defection, behaviour patterns that have evolved during the history of development of human behaviour. Ultimate behaviour analyses of these patterns do offer the opportunity to understand why humans behave in specific ways based on man's evolutionary origins and sources. Consequently, ultimate behaviour analyses could provide a solid foundation for the development of a framework giving insurers the possibility to exert influence on the insuree's behaviour and thus to assist in successfully impacting the moral hazard.

\section{Der moral hazard in der Versicherung}

Bei einer repräsentativen Umfrage gaben 76,6\% der Befragten an, dass es ihr gutes Recht sei, möglichst viel Geld von der Versicherung zurückzubekommen. Fast jeder Zweite hatte sogar den Willen, bei seiner Versicherung mindestens das herauszubekommen, was er einbezahlt (Meschkat und Nauert 2008). Diese Reaktion auf das Bezahlen der Versicherungsprämie kann als die Quelle des moral hazards, einer speziellen Form des subjektiven Risikos, angesehen werden (Mahr 1972). Der Begriff des Risikos bezeichnet die Gefahr der negativen Abweichung des tatsächlichen Ergebnisses vom erwarteten Ergebnis, d. h. die Gefahr des Eintretens einer ungünstigen, also nutzen- oder vermögensmindernden Entwicklung (Bühner 2001). In der Versicherungswissenschaft wird darunter ,die Möglichkeit des Schadeneintritts durch Verwirklichung einer versicherten Gefahr" verstanden (Fürstenwerth und Weiß 2001, S. 534). Risiken können objektiver und subjektiver Natur sein. Vom objektiven Risiko wird gesprochen, ,wenn die betrachteten unsicheren Werte objektiv, d. h. von einem beliebigen, normalen Betrachter wahrgenommen werden können. Ein subjektives Risiko liegt hingegen vor, wenn ein unsicherer Wert nur von dem jeweiligen Subjekt oder nur von einigen Subjekten wahrgenommen wird“ (Streitferdt 1973). Konkreter bezeichnet in der Versicherungswirtschaft das objektive Risiko „die Wahrscheinlichkeit dafür, dass ein bestimmtes versichertes Ereignis [...] eintritt, soweit diese 
Wahrscheinlichkeit von objektiv definierbaren mess- und quantifizierbaren Faktoren abhängt“" (Grossmann 1969, S. 68). Kann es allerdings durch ein Individuum, dessen Interessen von dem Risiko abhängen, zu einer Einflussausübung auf das Risiko kommen, wird vom subjektiven Risiko gesprochen.

Moral hazard (moralisches Risiko bzw. moralisches Wagnis) besteht in allen Bereichen, in denen ein Widerspruch zwischen Kollektivrationalität und Individualrationalität vorliegt. Ursache für das moralische Risiko ist die asymmetrische Informationsverteilung zwischen einer höheren und einer kollektiven Instanz. Bei Vertragsbeziehungen zur kollektiven Abdeckung von Risiken ergibt sich die Problematik, dass der Versicherer nicht überprüfen kann, ob der Versicherungsnehmer durch sein individuelles Verhalten Einfluss auf das Risiko nimmt. Der Versicherungsnehmer hat nach Abschluss eines Versicherungsvertrages einen Informationsvorsprung gegenüber dem Versicherungsunternehmen, der es ihm ermöglicht, ungerechtfertigte Ansprüche geltend zu machen (Meschkat und Nauert 2008). Diese Ansprüche ergeben sich aus dem nachlässigen Verhalten des Versicherungsnehmers gegenüber der versicherten Gefahr oder der aktiven Herbeiführung des Versicherungsfalls. Das moralische Risiko in der Versicherung bezeichnet demnach die ,mögliche Verhaltensänderung von Versicherungsnehmern nach Abschluss eines Versicherungsvertrages, die zu einer Erhöhung der Versicherungsleistungen führt und für den Versicherer nicht zu beobachten ist" (Nell 1998). Bedingt durch die Verhaltensänderung ist der erwartete Schaden der versicherten Gefahr, hinsichtlich Höhe und Eintrittswahrscheinlichkeit, mit Versicherungsschutz höher, als er unversichert wäre (Zweifel und Eisen 2003). Dies hat sowohl direkte Auswirkungen auf das Versicherungsunternehmen als auch indirekte Konsequenzen für die Versichertengemeinschaft. Während ein zu hoher Realschaden wirtschaftliche Schäden für den Versicherer bedeuten kann, können die zu hohen Schadenzahlungen zu einer Erhöhung der Risikoprämie des Gesamtkollektivs führen.

\section{Problematik und Zielstellung}

In der Ökonomie wird die Gefahr des moralischen Risikos und dessen Minimierung vorrangig in der Agency-Theorie der Neuen Institutionenökonomik behandelt. Die Neue Institutionenökonomik ist als Kritik an den Defiziten der neoklassischen Wirtschaftslehre und der ihr zugrunde liegenden Gleichgewichtstheorie entstanden. Das Idealbild wirtschaftlich agierender Individuen, auf dem die Neoklassik basiert, wird allgemein durch das theoretische Modell des homo oeconomicus abgebildet. Dessen Handeln ist durch fünf zentrale Elemente charakterisiert (Krol 1992): den methodologischen Individualismus, Rationalität, Trennung von Präferenzen und Restriktionen, die Existenz relevanter Alternativen und Eigennutz der handelnden Individuen. Zusätzlich wird indirekt eine unbeschränkte Informationsverarbeitungskapazität unterstellt (Söllner 2001). Die Prämissen der Neoklassik klammern viele der Probleme der realen Welt aus. Ein Teil der Lücken, die sich aus den Vereinfachungen der realweltlichen Zustände ergeben, können mit Hilfe von Ansätzen der Neuen Institutionenökonomik gefüllt werden. Die Grundlage bildet auch hier der (erweiterte) homo oeconomicus als heuristisches Menschenbild. Kernpunkt dieses 
Denkansatzes ist die Anerkennung von Koordinations- und Motivationsproblemen bei der Interaktion von Menschen in einer arbeitsteiligen Wirtschaft, zu deren Bewältigung Institutionen nötig sind (Göbel 2002). Unter einer Institution wird dabei ein Vertrags- oder Regelsystem einschließlich ihres Durchsetzungsmechanismus', durch welches das Verhalten von Individuen kanalisiert wird, verstanden (Erlei et al. 2007). Die Neue Institutionenökonomik besteht aus drei ineinander übergreifenden Ansätzen:

- die Prinzipal-Agent-Theorie zur Beschreibung, Erklärung und gegebenenfalls Gestaltung einer institutionellen Auftragsbeziehung durch entsprechende Vertragsgestaltungen,

- die Property-Rights-Theorie zur Erklärung und Gestaltung aller durchsetzbaren Verhaltensbeziehungen zwischen ökonomischen Akteuren,

- die Transaktionskostentheorie zur Berücksichtigung entstehender Kosten bei der Übertragung eines Gutes oder einer Leistung über eine technisch trennbare Schnittstelle hinweg.

Die Annahme positiver Transaktionskosten, wie es Grundgedanke der Neuen Institutionenökonomik ist, bedeutet, dass der Einzelne Zeit und Ressourcen zur Informationsbeschaffung aufwenden muss und dass er nur begrenzt in der Lage ist, diese Informationen $\mathrm{zu}$ verarbeiten. Positive Transaktionskosten führen demnach zu einer asymmetrischen Informationsverteilung, die die Ursache des moralischen Risikos darstellt. Die Auswirkungen der Informationsasymmetrie werden in der AgencyTheorie behandelt und Lösungsmöglichkeiten diskutiert. Die Beziehung zwischen einem Versicherer und dem Versicherungsnehmer bildet eine klassische PrincipalAgent-Relation. Zentrum der Agency-Theorie sind die vertraglichen Interaktionen zweier Parteien von Wirtschaftssubjekten, in diesem Fall das Versicherungsverhältnis zwischen dem Versicherungsnehmer und dem Versicherer. Die negativen Konsequenzen aus der asymmetrisch verteilten Information zwischen den beiden Marktteilnehmern können aus Sicht der Neuen Institutionenökonomik durch verschiedene Maßnahmen bekämpft werden (Göbel 2002). Dazu gehören die Reduktion der Informationsasymmetrie durch Monitoring und Reporting, die Verkleinerung des Interessenkonfliktes durch Bestrafungs- und Belohnungshandlungen sowie das Platzieren von Vertrauen.

Die Agency-Theorie bietet gegenüber dem Referenzmodell der neoklassischen Theorie zahlreiche Verfeinerungen. Bisher vernachlässigte Sachverhalte der Realität sind nun mit einbezogen. Dennoch lassen sich nicht alle Probleme lösen. Ein wesentlicher Kritikpunkt liegt in der Annahme des rationalen Handelns und der reinen Orientierung der Akteure an ihrem Eigeninteresse. Die Neue Institutionenökonomik fußt zwar auf dem Verhaltensmodell des erweiterten homo oeconomicus, grundlegende Prämissen wie die mittlerweile zwar eingeschränkte Rationalität und die Eigennutzannahme wurden jedoch beibehalten. Verhaltensökonomische Experimente haben gezeigt, dass diese Grundannahmen beim Menschen realitätsfremd sind (Fehr und Gächter 2000b) und dessen Entscheidungsverhalten systematisch von der Rationalität abweicht (Frey und Benz 2001). Darüber hinaus konnten deutliche Hinweise dafür erbracht werden, dass soziale Präferenzen wie Fairness oder Reziprozität im Verhalten der Menschen eine wesentliche Rolle spielen. 
Diese zahlreichen beobachteten Anomalien legen den Schluss nahe, dass ein Zurückgreifen auf den homo oeconomicus unzureichende Ergebnisse liefert. Es gelingt dem Modell nicht, menschliches Verhalten ausreichend präzise zu beschreiben (Kirchgässner 1991). Zur Untersuchung wirtschaftlicher Problematiken könnte daher ein verhaltensorientierter Ansatz der Realität näher kommen. Ein solcher Ansatz kann auf der Untersuchung der ultimaten Verhaltensursachen gründen. Die Betrachtung der ultimaten Verhaltensursachen des moralischen Risikos sollte ein realitätsnäheres Bild des wirtschaftlich handelnden Menschen liefern und ökonomische Erklärungsansätze in gewissem Maße erweitern können. Das moralische Risiko in der Versicherung steht im Spannungsfeld von kooperativen und defektionären Verhaltensweisen. Die Untersuchung der ultimaten Ursachen für kooperatives Verhalten könnte nun Rückschlüsse auf die Ursache des moralischen Risikos und darauf aufbauend mögliche Maßnahmen für Versicherer liefern.

\section{Die ultimaten Ursachen kooperativen Verhaltens}

\subsection{Die Soziobiologie zur Analyse ultimater Verhaltensursachen}

Charles Darwin hat bereits 1859 dargelegt, dass Verhaltensstrategien von Mensch und Tier ganz wie physische Merkmale auch, dem Prozess der natürlichen Selektion unterworfen sind. Erhöht eine bestimmte Verhaltensweise die individuellen Überlebensoder Fortpflanzungschancen, so wird diese sich langfristig innerhalb der Population durchsetzten. Der Anpassungswert einer Verhaltensstrategie läßt ich nicht a priori bestimmen, sondern ergibt sich aus der Interaktion des Verhaltens mit der Umwelt. Verhaltensstrategien, die heute beobachtet werden können, waren entweder in der Vergangeheit besser angepasst als andere oder haben sich in der Population gehalten, weil sie selektionsneutral waren, d. h. keinen Einfluss auf Überleben und Fortpflanzung ihres Trägers hatten.

Nach Tinbergen (1979) läßt sich Verhalten auf vier Ebenen evolutionsbiologisch erklären: Verhalten hat eine ontogentische Ursache, denn es ist irgendwann in der Individualentwicklung entstanden, es hat eine stammesgeschichtliche Ursache, denn nahe verwandte Arten sind sich gentisch und so auch im Verhalten ähnlicher, es hat eine proximate oder Wirkursache, denn hinter jeder Verhaltensäußerung eines Individuums steht eine physiologischer Mechanismus, und nicht zuletzt hat es eine ultimate oder Zweckursache, denn es ist durch den Prozess der natürlichen oder sexuellen Selektion hervorgebracht worden.

Die Benutzung des Begriffes Strategie impliziert hier nicht, dass Entscheidungen dem handelnden Individuum bewusst sind (Voland 2000). Vielmehr beschreibt der Begriff Strategie, den Zusammenhang zwischen dem beobachtbaren Verhalten und dessen Wirkursachen und macht den Anpassungswert somit messbar. Die Kosten einer Verhaltensstrategie können mit deren Nutzen hinsichtlich des Überlebens und der Fortpflanzung verrechnet und mit denen alternativer Strategien in der gegebenen Umwelt verglichen werden. Evolutionär stabil sind jene Strategien, die eindringenden Mutanten widerstehen und durch keine Alternativstrategie verbessert werden 
können (McFarland 1989). Im Extremfall können die dem Bewußtsein zugänglichen Beweggründe gar die Zweckursache verbergen, d.h., es kann adaptiv sein, dass dem Individuum nicht bewußt ist, in welcher Weise dessen Verhalten fitnessfördernd ist.

Die Soziobiologie ist eine Disziplin innerhalb der Verhaltensbiologie, die sich mit der Evolution von Sozialverhalten beschäftigt (Voland 2000). Andere Verhaltensaspekte sind etwa die Orientierung im Raum oder die Habitatwahl. Die Soziobiologie hat aber vielfache Berührunsgpunkte mit anderen Wissenschaftsdisziplinen etwa der Neurobiologie und der Ökologie (Wuketits 1997) und beschäftigt sich vornehmlich mit den ultimaten Ursachen sozialer Verhaltensäußerungen. Die Disziplin geriet in den 80er Jahren besonders außerhalb der Biologie in Kritik, als begonnen wurde soziobiologisches Denken auf menschliches Verhalten zu übertragen. Soziobiologen besprachen Aggression gegen Artgenossen bis hin zur Kindstötung oder Kriegsführung, die Angst vor dem Fremden und die Evolution von Geschlechterrollen, Themen also, die allesamt politisch aufgeladen sind. Ihnen wurde vorgeworfen, mit ihrem Paradigma eine Rechtfertigung für Ungleichheiten und Ungerechtigkeiten in menschlichen Gesellschaften zu schaffen. (Lewontin et al. 1984). Soziobiologische Forschung ist aber an sich wertfrei und ohne politische Zielsetzung (Wuketits 1997). Auch weisen Soziobiologen selbst immer wieder auf den naturalistsischen Fehlschluss hin: aus dem Sein kann nicht das Sollen abgeleitet werden. Wird beschrieben, wie Verhaltensstrategien evolutionär zustandegekommen sind, so heißt das nicht, dass sie deshalb unabänderlich oder gar gut, richtig und erstrebenswert sind. Winkler (1985) beschreibt die Kritik, die der Soziobiologie innerhalb der Biologie entgegengebracht wurde und faßt zusammen, dass sie hauptsächlich auf der Ablehnung der Individuen- oder Genselektion beruht. Die Kritiker sahen vielmehr die ultimate Ursache sozialer Verhaltensweisen im Vorteil für das Wohl der Gruppe oder in der Arterhaltung. Der zugrundligende Mechanismus der Gruppenselektion, wie er in den 70er Jahren propagiert wurde, ist jedoch nicht mit der Theorie der natürlichen Selektion zu vereinbaren (Winkler 1985).

Heute hat der Gedanke, dass menschliches Verhalten nicht beliebig kulturell formbar und vielfältig zu sein scheint auch außerhalb der akademischen Welt seinen festen Platz (Pease und Pease 2000). Es ist weithin anerkannt, dass Menschen sich in bestimmten sozialen Situationen überall auf der Welt ähnlich verhalten (Kotrschal 1995). Es scheint also lohnend, sich auf die Suche nach den Anpassungsund Selektionsvorteile zu machen, die solchen menschlichen Universalien zugrundeliegen. Sind diese verstanden, so lässt sich auch das Verhalten verstehen, vorhersagen und in gewissen Grenzen auch lenken oder verändern. Es muß jedoch ausdrücklich darauf hingewiesen werden, dass nicht alle menschlichen Verhaltensweisen vollständig adaptiv sind. Besonders beim Menschen haben Einflüsse aus dessen Umwelt einen starken Anteil an dessen Verhalten (Barash 1980). Auch soll die Auswirkung von Lernprozessen auf menschliches Handeln nicht bestritten werden. Vor der eingehenden wissenschaftlichen Untersuchung ist immer unsicher, welcher Anteil eines bestimmten Verhaltensmerkmales auf evolvierte Mechanismen zurückzuführen ist. Kann dieser Anteil jedoch identifiziert werden, so wird auch das Verhalten besser greifbar, verständlicher und letztendlich formbar. 


\subsection{Gruppenbildung als Grundlage sozialer Verhaltensweisen}

Primaten zeichnen sich unter den Wirbeltieren durch einen hohen Grad an Sozialität aus; sie leben überwiegend in recht stabilen Gruppen (Geissmann 2003). Aus soziobiologischer Sicht bilden sich Gruppen dann, wenn den Gruppenmitgliedern daraus ein Nutzen erwächst, der die individuellen Kosten des Gruppenlebens übersteigt. Primaten sind außerdem relativ langlebig (Kappeler und Pereira 2003). Daraus ergibt sich erstens, dass die Anzahl und Identität der Sozialpartner eines Primaten sehr beschränkt ist und zweitens, dass Individuen mit sehr großer Wahrscheinlichkeit vielfach und über einen langen Zeitraum hinweg miteinander interagieren. Aufgrund seiner engen Verwandtschaft zu den großen Menschaffen, ist es plausibel anzunehmen, dass auch der Mensch den Großteil seiner Jarhmillion dauernden Entwicklung in solchen stabilen Gruppen verbracht hat (Wuketitis 1997). Auch wenn heutzutage nur noch ein kleiner Teil der Weltbevölkerung in Kleingruppen lebt trägt der Mensch weiter Verhaltensstrategien in sich, die sich für das Leben in jenen Gruppen als vorteilhaft erwiesen haben. Das Ausbilden von Hierrachien, Streben nach Status, Kooperation und Defektion sollten vor diesem Hintergrund untersucht werden.

\subsection{Die Evolution kooperativer Verhaltensweisen}

\subsubsection{Ausprägungen kooperativen Verhaltens}

Kooperation ist die Interaktion zweier Individuen, welche dem Empfänger einen Vorteil einbringt und dem Akteur Kosten verursacht (West et al. 2007a). Kooperatives Verhalten setzt somit voraus, dass die Individuen in der Lage und motiviert sind, ihr Verhalten gleichzeitig und koordiniert auf ein gemeinsames Ziel hin auszurichten (Voland 2000). Im Idealfall führt Kooperation zu einem Nutzen aller Beteiligten (Wuketits 1997). Die einzelnen Individuen müssen zur Erreichung des gemeinsamen Ziels weniger Ressourcen einsetzen und tragen auch ein geringeres individuelles Risiko. Komplexe Kooperationsleistungen sind auch von Menschenaffen bekannt. Schimpansen gehen gemeinsam unter Einnahme komplementärer Rollen auf die Jagd und erreichen so Ziele, die bei solitärem Beutefang unerreichbar wären (Boesch 1994, Boesch et al. 2006). Auf diesem Erbe aufbauend hat sich die Kooperationsfähigkeit beim Menschen in besonderem Maße herausgebildet. Es scheint, als sei diese eng mit dem beim Menschen stark ausgeprägten prosozialen Verhalten (Burkart et al. 2007) verbunden, welches auch bei Primaten zu einem gewissen Grad beobachtbar ist (Warneken et al. 2007).

Kooperatives Verhalten kann nur dann evolutionsstabil sein, wenn es nicht nur einen Vorteil für den Empfänger der Transaktion bedeutet, sondern auch dem Initiator einen Nutzen bringt. Der Theorie der Individualselektion folgend, besitzt reiner Altruismus keine evolutive Chance (Trivers 1971). Individuen, die auf kooperatives Handeln verzichten, müssen die Kosten nicht tragen, können besser überleben und sich stärker fortpflanzen. Die von den Genen bereitgestellten kooperativen Verhaltensprogramme wären in der nächsten Generation nicht mehr in dem Maße präsent wie diejenigen der nicht-altruistischen Individuen. Das alternative altruistische Gen 
würde so langfristig aussterben. Kooperatives Verhalten kann in drei Gruppen klassifiziert werden (Krebs 1996): Mutualismus, Nepotismus und Reziprozität.

Folgt man Voland (2000), dann ist Mutualismus als kooperatives Verhalten zwischen Individuen zu verstehen, welches zum allseitigen Vorteil aller Beteiligten führt. Diese Verhaltensweise bezeichnet die Kooperation im eigentlichen Sinne. Das strategische Ziel des Mutualismus ist die Investition in ein gemeinsames Verhalten bei einer unmittelbaren Gewinnerwartung. Die Koordination des individuellen eigennützigen Verhaltens der Kooperationspartner fördert einen direkten gemeinschaftlichen Kooperationsgewinn. Für Mutualismus ist charakteristisch, dass mit Betrug oder Kooperationsverweigerung keine auch nur kurzfristigen Gewinnaussichten für das kooperationsverweigernde Individuum verbunden sind.

Nepotismus bezeichnet individuelle Fitnesskosten verursachende Verhaltensweisen, deren Effekt in der Fitnesssteigerung genetisch verwandter Individuen liegt. Trotz Nachteile für den Altruisten kann das altruistische Verhalten einen Vorteil für die verwandtschaftliche Reproduktion bedeuten. Der kostspielige Altruismus der uneigennützigen Individuen stellt im Grunde genommen nutzenbringenden Egoismus dar. Da sich die individuelle Fitness aus dessen eigenem genetischen Beitrag und dem seiner Verwandten zusammensetzt, bewirkt ein Individuum, welches die Fortpflanzungschancen eines nahen Verwandten erhöht, eine Steigerung der eigenen indirekten Fitness und somit seiner Gesamtfitness. Nepotistischer Altruismus ist durch die Hamilton-Ungleichung erklärbar (Voland 2000): $K<r \times N$. Es kann vorhergesagt werden, dass das Kosten-Nutzen-Verhältnis genau dem Verwandtschaftsgrad $r$ bzw. dem Anteil gemeinsamer Gene zwischen den Partnern entsprechen sollte, wenn die Kosten $K$ der Transaktion für den Akteur kleiner als der Nutzen $N$ für den Rezipienten ist. Nepotistisch-altruistisches Verhalten wird gemäß der Hamilton-Ungleichung um so wahrscheinlicher, je geringer die Nachteile für den Altruisten, je größer die Vorteile für den Empfänger und je enger Akteur und Empfänger miteinander verwandt sind. Nepotistisches Verhalten ist auch bei Primaten beobachtbar. Bei vielen Spezies der Altweltaffen bleiben die weiblichen Tiere ihr gesamtes Leben in der Gruppe, in welcher sie geboren wurden. Dort unterstützen sie nepotistisch ihre Schwestern, aber auch andere Verwandte ihrer Mutter (Langergraber et al. 2007). Bei nicht-menschlichen Primaten scheint Nepotismus die wichtigste Kooperationsform darzustellen und die teuersten Kooperationen ausschließlich den Verwandten vorbehalten zu sein (Silk 2006).

Kooperation ist auch dann zu beobachten, wenn der Verwandtschaftsgrad zwischen den Individuen gering ist oder gänzlich fehlt (Axelrod 2005). Kooperatives Verhalten wird beim Menschen meist durch einen anderen Mechanismus als den Nepotismus begünstigt: die Reziprozität zwischen unverwandten Individuen (Trivers 1971). Reziprozität bzw. reziproker Altruismus ist der anfängliche Verzicht auf die volle Ausschöpfung der persönlichen Reproduktionschancen zugunsten Dritter mit der späteren Belohnung durch die Erwiderung der Opferhandlung des ursprünglichen Empfängers (Voland 2000). Vereinfacht ausgedrückt handelt es sich dabei um die Wandlung egoistischer Verhaltensweisen in altruistische Handlungen. Reziproker Altruismus kann sich nur herausbilden, wenn der Altruist aus seinem Verhalten einen Eigengewinn ziehen kann, der in Bezug auf dessen Gesamtfitnesseignung größer als die Kosten ist (Barash 1980). Der Gewinn für den Altruisten muss die mit 
der altruistischen Anfangshandlung verbundene Minderung der Gesamteignung übersteigen. Das reziprok-altruistische Verhalten ist für den Altruisten mit einem geringen Risiko, aber für den Hilfeempfänger mit einem großen Nutzen verbunden, nach Barash (1980) eine Grundvoraussetzung für die Herausbildung von wechselseitigem Altruismus.

Reziproke Verhaltensweisen sind auch bei Primaten, besonders bei Menschenaffen und Schimpansen bekannt (Voland 2000). Beobachtungen haben gezeigt, dass nichtverwandte Schimpansen reziprok Fellpflege betreiben und Fellpflege gegen Nahrung eintauschen oder mit Unterstützung in Streitsituationen entgelten (Schino 2007). Es wird davon ausgegangen, dass direkte Fitnessgewinne Schimpansen zu diesen kooperativen Verhaltensweisen veranlassen (Langergraber et al. 2007). Am ehesten kommt das Prinzip der Gegenseitigkeit bei intelligenten Arten mit einem ausgeprägten Sozialgefüge vor (Barash 1980). Besonders beim Menschen stellt die Reziprozität ein weitverbreitetes Muster sozialen Verhaltens dar (Immelmann 1988, Rabin 2002).

Mit dem reziproken Altruismus hat sich bei höheren Lebewesen ein weiteres Phänomen kooperativen Verhaltens ausgebreitet: die indirekte Reziprozität. Bei der indirekten Reziprozität handelt es sich um eine besondere Form des Austauschs (Stegbauer 2002). Die gegenseitigen Leistungen lassen sich nicht direkt verrechnen oder durch zeitlich nah beieinander liegende Tauschakte in Verbindung setzen. Indirekte Reziprozität ist demnach eine Form der Reziprozität, bei der eine Hilfeleistung zu einem späteren Zeitpunkt von einem anderen Individuum erwidert wird, das aber nicht der Empfänger der ursprünglichen Leistung war (Dufwenberg 2000). In diesem Sinne kann die gemeinsame Gefahrentragung innerhalb einer Versichertengemeinschaft auch als reziproker Altruismus interpretiert werden.

\subsubsection{Die Gefahr ausnützerischer Verhaltensweisen}

Kooperatives Verhalten ist immer durch einseitiges ausnützerisches Verhalten bedroht. Dieses ausnützerische Verhalten soll im Folgenden als Defektion bezeichnet werden. Wie in den vorhergehenden Kapiteln ausgeführt, spielt Betrug bei mutualistischem und nepotistischem Verhalten eine untergeordnete bis gar keine Rolle. Es ist vielmehr der reziproke Altruismus, der für den Initialdonor die Gefahr des Betruges mit sich bringt (Barash 1980). Das Problem besteht im Wesentlichen darin, dass ein Individuum durch Reziprozität zwar profitieren kann, es jedoch mehr gewinnt, wenn es die kooperativen Anstrengungen anderer ausnutzt (Krebs 1996). Da im Normalfall nicht simultan reziproziert wird, muss ein Partner immer eine Vorschussleistung erbringen und darauf hoffen, dass der Rezipient diese irgendwann zurückzahlt. Die Situation ist mit dem in der Spieltheorie als Grundproblem bekannten Gefangenendilemma vergleichbar (Axelrod 2005, Immelmann 1988). Bei beiden Interaktionspartnern würde sich das unkooperative Verhalten als dominante Strategie ergeben und beidseitige Defektion ein Nash-Gleichgewicht bilden, also eine Situation, bei der kein einzelner Spieler für sich einen Vorteil erzielen kann, indem er einseitig von seiner Strategie abweicht. Es ist weiter davon auszugehen, dass das individuelle Verhalten der Individuen an die nächste Generation vererbt und der geschilderte Konflikt in den Folgegenerationen wieder auftauchen wird. Reziprok-kooperatives Verhalten kann in diesem Fall nicht evolutionär stabil sein. In einer Population kooperieren- 
der Tiere würde ein neu auftretender defektierender Verhaltensmutant besonders gute Fortpflanzungserfolge erzielen. Es bestünde dadurch ein Selektionsvorteil des $\mathrm{Mu}-$ tanten, der sich daraufhin stärker vermehren und seinen zahlenmäßigen Anteil in der Population Generation für Generation erhöhen könnte. Das unkooperative Verhalten würde die einzig evolutionär stabile Strategie für diese Situation darstellen (Immelmann 1988). Sind erst einmal die Mitglieder der Population Träger dieses erblichen Merkmals, so besitzt jede Mutationsstrategie einen Selektionsnachteil. In einer Population permanenter Defektionäre könnte eine Mutation zu kooperativen Verhalten niemals vorteilhaft sein.

Reziprozität ist nicht nur beim Menschen beobachtbar. Tiere tauschen ganz unterschiedliche Ressourcen und Dienstleistungen gegeneinander aus, manchmal sogar zwischen Arten. Diese Verhaltensform konnte bei unterschiedlichen Spezies beobachtet werden (Packer 1977, Fischer 1980). Es muss also davon ausgegangen werden, dass sich in der Evolutionsgeschichte Mechanismen entwickelt haben, die zu einer Eindämmung von Defektion geführt haben.

\subsubsection{Voraussetzungen für die Evolution kooperativer Verhaltensweisen}

Defektion ergibt sich in der biologischen Evolution als Lösung für ein einmaliges Treffen von Interaktionspartnern (Axelrod 2005). Wenn die Auszahlung in Graden an Fitness erfolgt und die Interaktionen zwischen Paaren von Individuen zufällig und nicht wiederholt sind, dann entwickelt sich jede Population zu einem Zustand, in dem alle Individuen defektieren. Wie aufgezeigt, ist die Strategie der Defektion evolutionär stabil, unter der Voraussetzung, dass die Spieler nicht erneut aufeinander treffen. In vielen biologischen Zusammenhängen haben die Paare von Individuen allerdings häufiger miteinander zu tun, was die Kontinuität reziprok-altruistischen Verhaltens begünstigt (Voland 2000). Wichtig dabei ist, dass die Interaktionszahl für die Individuen nicht absehbar ist. Wenn es zu einer bekannten Zahl von Interaktionen zwischen den Paaren kommt, ist Defektion die einzig evolutionär stabile Strategie (Axelrod 2005). Damit eine häufige Kooperation zwischen den Individuen zustande kommt, muss es eine hohe Wahrscheinlichkeit geben, dass beide Partner wieder aufeinander treffen.

Wie bereits dargestellt wurde, ist die Voraussetzung dafür, dass die Zukunft für die Interaktionspartner relativ zur Gegenwart hinreichend von Bedeutung ist. Dies ist nur möglich, wenn die Akteure ohne eine Begrenzung in der Zukunft weiter aufeinander treffen und sie sich wiedererkennen. Wie eingangs bemerkt, hat sich der Mensch im Laufe seiner Evolutionsgeschichte als soziales Wesen entwickelt. Gruppenbildung begünstigt Kooperation, da sie jedem Mitglied eine nicht triviale Wahrscheinlichkeit gibt, auf ein kooperatives Individuum zu treffen. Die Gruppengröße $n$ hat einen direkten Einfluss auf die Wahrscheinlichkeit $p$ des erneuten Aufeinandertreffens zweier bereits bekannter Individuen. Im einfachsten Fall ist dies $p=1 / n$.

Direkte Reziprozität kann nur erfolgreich sein, wenn Individuen ihre Interaktionspartner wiedererkennen und sich an das Ergebnis der letzten Begegnung erinnern können (Dugatkin 2002). Höhere Grade an Kooperation werden erreicht, wenn dieselben Partner wiederholt zusammenkommen und jedem das Verhalten der anderen aus vorangegangenen Transaktionen bekannt ist (Semmann et al. 2005). Die Fähig- 
keit zur Diskriminierung, also zur Wiedererkennung, Unterscheidung und daraus folgenden Ungleichbehandlung, erlaubt es, Interaktionen mit verschiedenen Individuen durchzuführen, ohne diese Individuen alle gleich zu behandeln. Direkte Reziprozität setzt demnach besondere kognitive Fähigkeiten der interagierenden Individuen voraus (Milinski 1998). Beim Menschen geschieht die Diskriminierung der Interaktionspartner durch die Erkennung und Erinnerung von Gesichtern (Axelrod 2005). Die Bedeutung, die die biologische Evolution des Menschen dieser Fähigkeit zuteilkommen ließ, macht die Gehirnstörung Prosopagnosie sichtbar. Die Betroffenen haben aufgrund von Läsionen in einer Region, die sich von Hirnhauts- bis Schläfenlappen hinzieht, Schwierigkeiten, Menschen anhand ihrer Gesichtszüge zu identifizieren. Die Lokalisation der Ursachen und der spezifisch durch sie ausgelöste Effekt machen laut Geschwind (1979) deutlich, dass die Wiedererkennung von Gesichtern eine hinreichend wichtige Aufgabe ist, um einen bedeutsamen Teil der Ressourcen des Gehirns dafür einzusetzen.

Primaten besitzen kognitive Fähigkeiten, die sich sehr gut zur Generierung sozialer Informationen eignen. Beispielsweise sind sie in der Lage, Individuen wiederzuerkennen, Verwandte zu identifizieren, den Wert von Diensten zu berechnen, vergangene Interaktionen mit Gruppenmitgliedern zu erinnern und zwischen kooperierenden und defektierenden Individuen zu unterscheiden (Silk 2007). In diesem Zusammenhang wird auch von dem sozialen Gehirn (social brain) gesprochen. Die kognitive Fähigkeit, mit Individuen soziale Beziehungen einzugehen, hat allerdings eine natürliche Grenze. Diese Grenze wird als Dunbar-Zahl bezeichnet. Dunbar (1998) geht davon aus, dass ein direkter Zusammenhang zwischen dem Gehirnvolumen von Säugern und der maximalen Gruppengröße, in welcher diese leben, besteht. Die Verarbeitungsleistung des Gehirns gibt demnach die kognitive Grenze für die Größe sozialer Gruppen vor. Daraus lässt sich schließen: Je größer oder komplexer strukturiert das Gehirn ist, desto stabilere, intensivere und zahlreichere Beziehungen können aufrechterhalten werden. Wird dieser Zusammenhang auf die Hirngröße des Menschen projiziert, der sich durch relativ große Neocortexbereiche im Gehirn auszeichnet, so lässt sich eine soziale Gruppengröße von etwa 150 vorhersagen. Menschen scheinen sich und ihre Welt um diese Zahl herum zu organisieren. In vorindustriellen Gesellschaften überall auf der Welt bildeten sich Clans mit ungefähr 150 Mitgliedern, und im heutigen England pflegen Menschen zu etwa 150 Individuen engeren persönlichen Kontakt (Hill und Dunbar 2003). Dunbar et al. (1999) sehen einen direkten Zusammenhang zwischen der Gruppengröße und dem Trittbrettfahrertum. Sie führen weiter aus, dass dieses Trittbrettfahrertum überhaupt nur in großen Gruppen besteht, da in kleinen Gruppen ein individuelles Kennen dies verhindert. Gruppenbildung hat sich demnach als evolutionär stabile Strategie mit der Folge einer Kooperation der einzelnen Teilnehmer etabliert. Sobald die Gruppengröße allerdings eine bestimmte Zahl überschreitet, führt dies, aufgrund der minimalen Wahrscheinlichkeit des erneuten Aufeinandertreffens und der mangelnden kognitiven Wahrnehmung, zu einer Begünstigung ausnützerischen Verhaltens.

Mit der Fähigkeit zur Diskriminierung als grundlegender Voraussetzung für die Evolution von Kooperation geht der Aufbau von Reputation einher. Reputation soll hier als Gesamtheit dessen verstanden werden, wie ein Individuum von seiner sozialen Gruppe unter Einbezug vergangener Aspekte wahrgenommen wird. Kooperation 
kann evolvieren, wenn Individuen vorzugsweise mit jenen Subjekten agieren, die in der Vergangenheit kooperative Verhaltensweisen gezeigt haben (Röhl et al. 2007). Dies ist besonders bei der Entwicklung indirekter Reziprozität von Bedeutung. Der Akteur wird nicht vom Rezipienten direkt entlohnt, sondern von einer dritten Partei. Die dritte Partei entscheidet unter anderem anhand der individuellen Reputation. Individuen, die eine Geschichte des Gebens hinter sich haben, werden vorzugsweise Empfänger der Kooperationsleistung (Semmann et al. 2005).

Experimente lassen erkennen, dass sich indirekte Reziprozität durch Reputation verbessern und gegen Betrug resistent werden kann (Röhl et al. 2007). Diskriminierende Strategien, bei welchen altruistisches Verhalten eine verbesserte und Egoismus eine verschlechterte Reputation zur Folge hat, können sogar zu einer stabil reziproken Population führen (Nowak und Sigmund 1998). Die Logik, die dem adaptiven Wert der indirekten Reziprozität in diesem Fall zugrunde liegt, ähnelt der Logik, auf welche die in der Spieltheorie bekannte streng-reziproke Strategie Tit-for-Tat aufbaut (Axelrod 2005): Egoismus erzeugt Egoismus und Kooperation erzeugt Kooperation, allerdings über dritte Parteien (Krebs 2000). Studien, unter anderem von Leimar und Hammerstein (2001), Panchanathan und Boyd (2003) und Nowak und Sigmund (1998), haben gezeigt, dass indirekte Reziprozität durch Reputation evolutionär stabil werden kann. Die Bedeutung des Ansehens für die Entwicklung der Kooperation wird auch durch Alexander (1987) erkannt, der in der sozialen Reputation die Ursache für die Evolution indirekter Reziprozität sieht und diese als die biologische Basis menschlicher Moralität definiert.

Kooperation kann auch durch die Minimierung des Interessenkonflikts gefördert werden. Reziproker Altruismus ist umso wahrscheinlicher, je kostspieliger es für den potenziellen Betrüger wird, Nutzen in Empfang zu nehmen, aber selbst nicht altruistisch zu handeln (Voland 2000). Wenn die Bestrafung für Defektion so groß ist, dass Kooperation unabhängig vom Verhalten des Interaktionspartners die beste Entscheidung ist, dann gibt es kein Dilemma mehr (Axelrod 2005). Bestrafungsverhalten als Form der Kooperationsförderung ist auch bei Primaten beobachtbar. Während des Nahrungsaustauschs bei Schimpansen treten selten Aggressionen auf. Kommt es aber zu einem solchen Verhalten, sind die Opfer meist die wenig kooperativ handelnden Gruppenmitglieder, also jene, die vermehrt Nahrung empfangen, ohne ihrerseits zu teilen (Voland 2000). Zwischen der Aggression und dem auslösenden Verhalten kann dabei eine teils beachtliche Zeitspanne liegen. Es ist daher davon auszugehen, dass Schimpansen über ein soziales Langzeitgedächtnis verfügen, in welchem soziale Transaktionen dauerhaft gespeichert werden können. Es ist anzunehmen, dass das Gedächtnis ähnlich dem des Menschen arbeitet, welches in der Lage ist, soziale Einseitigkeiten aufzuspüren und die Abweichung von sozialen Regeln wie der Reziprozität als Regelverletzungen zu erkennen. Das aggressive Verhalten der Schimpansen lässt sich als eine Sperre interpretieren, die verhindert, dass es leicht zu einer Akkumulation einseitiger Vorteilsnahme durch Ausbeutung individueller Beziehungen kommen kann. Das Verhalten bei der Nahrungsteilung des Schimpansen ist von besonderem Interesse, da dies als die Grundvoraussetzung für die Evolution von Arbeitsteilung in der Stammlinie der Hominini angesehen wird (Henke 1999).

Die Verminderung des Interessenkonflikts kann mittels Belohnung für kooperatives Verhalten oder Bestrafung bei Opportunismus stattfinden. Bei der Entwicklung 
der Kooperation beim Menschen scheint die Bestrafung der Defektion eine besondere Rolle gespielt zu haben. Die Auswirkung von Bestrafungsmöglichkeiten auf die Kooperationsbereitschaft des Menschen sind experimentell bestätigt worden (West et al. 2007b). Der Mensch scheint lieber zu bestrafen, als dass er belohnt. Dieses Ergebnis wird auch durch neurobiologische Erkenntnisse gestützt. Experimente haben gezeigt, dass die Bestrafung von unkooperativen Individuen zu einer Stimulation im dorsal striatum, einer Gegend im Belohnungszentrum des Gehirns, führt. Proximat betrachtet bestrafen Individuen unkooperatives Verhalten demnach, weil es ihnen ein Gefühl der Befriedigung gibt. Das erlaubt die ultimate Interpretation, dass sich das Gehirn in dieser Weise entwickelt hat, weil die Bestrafung einen direkten oder indirekten Fitnessgewinn für das bestrafende Individuum mit sich führt (West et al. 2007a).

Der Mensch ist sogar bereit, Opfer für die Bestrafung unkooperativer Individuen zu bringen (Camerer et al. 2004). Ein Individuum, welches auf eigene Kosten ohne einen direkten Nutzen das unkooperative Verhalten eines anderen Individuums bestraft, wird als starker Reziprokator bezeichnet (Gintis 2000). Starke Reziprozität selbst bei anonymen Interaktionspartnern und deren positive Auswirkung auf kooperative Verhaltensweisen wurden in vielen Experimenten beobachtet (Fehr et al. 2002). Tucker und Ferson (2008) schließen daraus, dass die natürliche Auslese den Menschen so geformt hat, dass er eine besondere Art des Eigeninteresses verfolgt, welches Fairness, Gleichheit und Gerechtigkeit sowie die Angst vor Betrug beinhaltet. Obwohl die Bestrafung von Egoisten sich als kooperationsfördernd herausgestellt hat, betonen Fehr und Rockenbach (2003) auch deren mögliche negative Effekte. Bestrafung ist nur wirksam zur Förderung von Kooperation, wenn sie sozial gerechtfertigt ist, also nicht zur Durchsetzung eines höheren materiellen Ergebnisses für den Bestrafenden führt. Wenn sozial gerechtfertigt, hat sich beim Menschen die Bestrafung unkooperativer Individuen als erfolgreich zur Evolution kooperativer Verhaltensformen erwiesen. Dieser Prozess geht sogar so weit, dass menschliche Individuen Betrüger nicht nur für etwas bestrafen, was sie ihnen angetan haben, sondern auch für etwas, was anderen Individuen angetan wurde (Fehr und Fischbacher 2004).

Förderung der Kooperation in einer Gesellschaft besteht häufig darin, die Individuen dazu zu bewegen, sich um das Wohlergehen anderer zu sorgen. Die Beeinflussung zur Verhaltensänderung findet häufig durch Eltern statt (Axelrod 2005), die die Werte der Kinder so formen, dass deren Präferenzen nicht nur die eigene Wohlfahrt, sondern auch in einem gewissen Grade die Wohlfahrt anderer sichern. Diese Form der Manipulation betrifft in einer soziobiologischen Sichtweise vorrangig die Förderung des nepotistischen Altruismus (Voland 2000). Dieser kann auch auf einfache Art und Weise unabhängig von nepotistischen Überlegungen stattfinden. Der einfachste Weg, kooperatives Verhalten zu erzeugen, ist die Belehrung über die Vorteile reziproker Verhaltensweisen (Axelrod 2005). Semmann et al. (2003) haben experimentell gezeigt, dass die freie Entscheidung zur Teilnahme an einer Kooperationsbeziehung Einfluss auf das Maß an kooperativen und defektionären Verhalten hat. Besteht die freie Wahl zur kooperativen Interaktion mit anderen Individuen, kommt es zu weniger Defektionen. Die Manipulation zur freiwilligen Kooperation kann und konnte sich demnach als effektive Maßnahme zur Förderung kooperativen Verhaltens erweisen. 


\section{Die Anwendung der Kenntnisse der ultimaten Verhaltensanalyse auf den moral hazard}

\subsection{Soziobiologische Sicht auf Versicherung und moral hazard}

Wie erläutert, hat sich die Bildung von Gruppen bei den Menschen über Jahrmillionen hinweg als evolutionär stabile Strategie etabliert. Die Kosten, die mit der Ansammlung in Sozietäten verbunden waren, mussten immer niedriger als der mit ihnen verbundene Nutzen sein. Ähnlich verhält es sich bei der Versicherung. Das Prinzip der kollektiven Selbsthilfe ist tief in der Geschichte des modernen Menschen verankert. Bereits aus dem zweiten Jahrtausend vor Christus ist bekannt, dass sich Karawanenteilnehmer aus Babylonien gegenseitig verpflichteten, Schäden gemeinsam zu ersetzen, die einem Teilnehmer dieser Reisegesellschaften entstanden (Zedtwitz 1999).

Was als zweckdienliches Verhalten einer kleinen Gruppe begann, hat sich im Laufe der Jahrhunderte als erfolgreiches System entwickelt. Mittlerweile haben sich allein in Deutschland die Bürger mit über 430 Millionen Versicherungsverträgen abgesichert (GDV 2008a). Das bedeutet, dass jeder Bundesbürger vom Kleinkind beginnend fast sechs Policen sein Eigen nennen kann. Ein solcher Erfolg des Prinzips Versicherung konnte nur möglich sein, weil der Nutzen die Kosten der Unternehmung im Mittel kontinuierlich überstieg. Das Prinzip Versicherung hat sich demnach ähnlich der Gruppenbildung als evolutionär stabil erwiesen. Die Versicherung kann dabei als Gruppe angesehen werden, welche aus dem Kollektiv und dem Versicherer besteht. Im Allgemeinen ist dem versicherten Individuum nur der Versicherer selbst bzw. ein für ihn tätiger Mitarbeiter bekannt und er nimmt diesen als Interaktionspartner wahr.

Mit der Bildung der Versicherungskollektive kommen auch die aus der frühgeschichtlichen Gruppenbildung des Menschen entstandenen Verhaltensmuster zum Vorschein: kooperatives Verhalten und die Gefahr der Defektion. Reziprozität wurde als wichtige Form der Kooperation zwischen nichtverwandten Individuen angeführt. Das Prinzip der Reziprozität kommt auch bei der Versicherung zum Ausdruck. Das Individuum schließt eine Versicherung ab und bekommt als Gegenleistung von dem Versicherer Versicherungsschutz. Das Versicherungsunternehmen übernimmt als Erwiderung der Prämienzahlung das definierte Risiko des Versicherungsnehmers (Farny 2006). Das Problem kann nun darin liegen, dass das Individuum den Versicherungsschutz gar nicht als direkte Gegenleistung für seine Vorleistung wahrnimmt. Der Versicherungsnehmer will für seine Geldzahlung einen sichtbaren Gegenwert. Er erwartet, auch ohne dass ein Schaden eingetreten ist, eine Zahlung des Versicherungsunternehmens (Theil 2002). Dieses Phänomen wurde experimentell nachgewiesen und unter der Bezeichnung Versicherung als Investition charakterisiert. Das Nichteintreten eines Schadens und damit auch das Fehlen einer Zahlung durch den Versicherer werden im Verhältnis zur gezahlten Prämie des Versicherungsnehmers als ungerecht empfunden. Um subjektive Gerechtigkeit wiederherzustellen (Fetchenhauer 1998), wird der Versicherungsnehmer vermehrt Versicherungsleistungen in Anspruch nehmen und aus Sicht des Versicherers defektieren.

Wenn Versicherung und das moralische Risiko unter dem Gesichtspunkt evolvierter Reziprozität aufgefasst werden, so könnten in der Evolutionsgeschichte 
etablierte Strategien und Faktoren, die die Entwicklung kooperativer Verhaltensweisen beim Menschen ermöglicht haben, erfolgsverspechende Gegenmaßnahmen gegen defektionäres Verhalten bilden. Das scheint umso wichtiger, da klassische vertragliche Maßnahmen wie Selbstbehalte in der Versicherungspraxis grundlegend nicht als Instrumente zur Steuerung des moralischen Risikos verstanden werden können (Schwarze 1997).

Die wesentlichen Erkenntnisse, die aus der ultimaten Verhaltensanalyse gezogen werden können, sind zum einen, dass dem Menschen gewisse Verhaltensweisen aufgrund seines evolutionären Erbes inhärent sind, und zum anderen, dass es in der Evolution der Spezies Mensch gewisse Umstände gab, die es dem Menschen erst ermöglichte, diese Verhaltensweisen zu entwickeln. Dazu gehören die Reziprozität des Menschen, begünstigt durch eine hinreichend hohe Wahrscheinlichkeit zur erneuten Interaktion, die Fähigkeit zur Diskriminierung des Gegenübers, die Möglichkeit zur Verminderung des Interessenkonflikts sowie den Aufbau von Reputation. Auf diesen Kenntnissen gründend, können diese Mechanismen unter Berücksichtigung der dem Menschen inhärenten Verhaltensweisen ein Rahmenwerk bilden, das den moral hazard effektiv bekämpfen könnte. Die Maßnahmen eines solchen ganzheitlichen Ansatzes betreffen in besonderem Maße die Prozessgestaltung des Versicherungsunternehmens und dessen Absatzpolitik.

\subsection{Verbesserung der Fähigkeit zur Diskrimination als Anforderung an die Prozessgestaltung}

Kooperation kann sich als Verhaltensmuster nur etablieren, wenn die Zukunft relativ zur Gegenwart hinreichend wichtig ist. Grundvoraussetzung dafür ist die Wiedererkennung des Interaktionspartners und die Erinnerung seiner in der Vergangenheit getätigten Transaktionen. Was bei Menschen und Primaten über Gesichtserkennung und das soziale Gehirn funktioniert, muss bei Institutionen über andere Mechanismen erreicht werden. Maßnahmen zur Diskriminierung der Versicherungsnehmer haben daher generellen Einfluss auf die gesamte Prozessgestaltung, insbesondere aber auf den Vertriebsprozess sowie den Vertrags- und Schadenbearbeitungsprozess.

Da die grundsätzliche Ursache für das moralische Risiko in der asymmetrischen Informationsverteilung zwischen dem Versicher und dem Versicherungsnehmer liegt, stellt es für das Unternehmen eine große Hürde dar, ausnützerisches Verhalten des Versicherten zu erkennen. Durch die Diskriminierung des Interaktionspartners und der Erinnerung vergangener Transaktionen besteht die Möglichkeit, auf zukünftiges Handeln zu schließen. Auffälliges Verhalten, z. B. häufige Schadenansprüche oder hohe Schadenzahlungen in der Vergangenheit, können bei gegenwärtigen Interaktionen den Schluss auf für den Versicherer und das Kollektiv schadhaftes Verhalten nahelegen und so ggf. zu einer Ablehnung von Ansprüchen führen oder Folgemaßnahmen auslösen.

Ein solches künstliches soziales Gehirn ließe sich, wie teilweise schon in speziellen Software-Anwendungen zur Betrugserkennung geschehen, auf technischem Wege realisieren. Eine Datenbank mit relevanten Kundendaten existiert bereits in jedem Versicherungsunternehmen. Zusätzlich sollten aber noch das Kundenverhalten und die Erfahrungswerte der Mitarbeiter mit direktem Kundenkontakt hinterlegt wer- 
den, um so ein ganzheitliches Datawarehouse zu erzeugen, das nach dem Vorbild der Evolution ein soziales Gedächtnis darstellt. Dieses Datawarehouse sollte im Vorfeld der Schadenprüfung abgefragt und auch mit den Erkenntnissen ex post gepflegt werden. Der so gewonnene Datenbestand kann nun in Folgeprozessen systematisch analysiert werden. Die Gesamtheit dieser Verfahren wird als Business Intelligence bezeichnet, deren zentrale Komponente das Datawarehouse darstellt (Bahlinger 2004). Business Intelligence ermöglicht es dem Versicherungsunternehmen, die Daten aus den heterogenen Quellen zu verarbeiten und mittels Analysewerkzeuge eine effektive Auswertung des multidimensionalen Datenbestandes durchzuführen (Pirk und Wolf 2001). Der Einsatz im Betrugsmanagement ermöglicht es, bestimmte Muster zu erkennen und so betrügerisches Verhalten zu identifizieren. Dabei kann Software aus dem Bereich der künstlichen Intelligenz und neuronaler Netze genutzt werden und diese können noch um Visualisierungswerkzeuge ergänzt werden.

Die intern erhobenen Daten zeigen aber nur einen Ausschnitt aus dem Verhalten des Versicherungsnehmers. Zur Erzeugung und Analyse eines vollständigen Verhaltensbildes werden noch Informationen aus Transaktionen mit anderen Versicherungsunternehmen benötigt. Die im Gesamtverband der deutschen Versicherungswirtschaft (GDV) organisierten Versicherungsunternehmen unterhalten die gemeinsame Warnund Hinweisdatei Uniwagnis. Das Informationssystem dient zur Prüfung im Leistungsfall bei den Versicherungen sowie insbesondere zur Aufdeckung und Prävention von Versicherungsbetrug und Versicherungsmissbrauch (GDV 2008b). Dabei werden Warndateien nach einem einheitlichen Verfahren informationstechnisch separat organisiert. Nimmt ein Unternehmen an Uniwagnis teil, erfolgt im Schaden- bzw. Leistungsfall in Bezug auf den einzelnen Versicherungsnehmer oder eine dritte Person hinsichtlich des konkreten Ereignisses eine Punktevergabe, die sich an vordefinierten unterschiedlich gewichteten Kriterien orientiert. Wird bei einem konkreten Versicherungsfall eine bestimmte Punktzahl durch den Versicherten überschritten, wird dies Uniwagnis gemeldet. Die in Uniwagnis abgelegten Daten können nun im Verlauf einer Antrags- oder Leistungsprüfung abgefragt und so bereits auffällig gewordene Versicherungsnehmer wiedererkannt werden.

Abwicklungsprozesse, die auf eine Business Intelligence - Architektur mit einem erweiterten internen Datawarehouse als zentralem Element und ein unternehmensübergreifendes zentrales Register zurückgreifen, können dem Versicherer die Möglichkeit bieten, die einzelnen Individuen des Kollektivs und deren Transaktionsgeschichte zu diskriminieren. In der Evolution hat sich diese Fähigkeit als Grundlage zur Entwicklung kooperativen Verhaltens erwiesen. Als ähnlich vorteilhaft sollte sie sich auch für das Versicherungsunternehmen erweisen können. Selbstverständlich ist bei all diesen Aktionen den Regelungen des Datenschutzes Rechnung zu tragen.

\subsection{Mögliche Maßnahmen der Absatzpolitik}

\subsubsection{Absatzprogrammpolitik}

Die Absatzpolitik bezeichnet „die Gestaltung der Absatzfunktion durch Festlegung von Absatzzielen und den Einsatz [geeigneter] Instrumente“ (Farny 2006, S. 673). Integraler Bestandteil der Absatzpolitik ist die Absatzprogrammpolitik. Darunter wer- 
den die Gestaltung der vom Versicherungsunternehmen angebotenen Produkte und deren Zusammenfassung zu einem Sortiment verstanden. Bestandteile der Absatzprogrammpolitik sind die Produktgestaltungspolitik und die Sortimentspolitik, in welchen es gilt, Maßnahmen zur Minderung des moral hazards zu etablieren.

Besonders bei Haftpflichtversicherungen besteht ein großer Zusammenhang zwischen dem Verhalten des Versicherungsnehmers und dem Versicherungsschaden. Das wird auch durch die Bezeichnung insurance against carelessness deutlich (Parsons 2003). Im Zuge der evolutionären Verhaltensanalyse konnte gezeigt werden, dass der Mensch ein reziprokes Wesen ist und sich kooperative Verhaltensweisen nur entwickeln konnten, wenn die Wahrscheinlichkeit der erneuten Interaktion hinreichend hoch ist. Auf dem deutschen Versicherungsmarkt stehen 609 Versicherungsunternehmen unter Bundesaufsicht (GDV 2008a). Davon sind 143 Versicherungsunternehmen in der allgemeinen Haftpflichtversicherung aktiv. Das heißt, die Wahrscheinlichkeit des erneuten Aufeinandertreffens des Versicherten auf das Versicherungsunternehmen nach Ablauf des Vertrages beträgt nur ca. $0,7 \%$ und würde bedeuten, dass die Zukunft relativ zur Gegenwart für den Versicherten einen untergeordneten Stellenwert besitzt und somit die Motivation zur Defektion sehr hoch ist. Tatsächlich gibt es Indizien für einen Zusammenhang von Vertragsdauer und Schadenquote (Alpar 2000).

Im Normalfall ist die Entscheidung für einen Versicherer nicht zufallsabhängig. Vielmehr ist sie die Konsequenz der Abwägung individueller Präferenzen. Ziel des Versicherers sollte es daher sein, mit Hilfe des Absatzprogrammes langfristige Bindungen mit den Versicherten zu etablieren, um so die Wahrscheinlichkeit erneuter Interaktionen signifikant zu steigern. Eine solche Bindung kann durch Verbundeffekte, also durch das Nutzen möglicher Cross- oder Up-Selling-Potentiale, erreicht werden (Wagner und Warmuth 2005). Maßgebliche Voraussetzung dafür wäre ein besonders breites Sortiment an Versicherungs-, aber auch an Kapitalanlage- und sonstigen Produkten.

Zentrales Instrument der Absatzpolitik ist eine an den Kundenbedürfnissen orientierte Produktgestaltung. Diese dient dem Aufbau langfristiger Beziehungen und stellt somit, gemäß den evolutionären Kenntnissen über die Entwicklung kooperativen Verhaltens, eine Grundvoraussetzung zur Minderung defektionären Verhaltens dar. Tatsächlich kann festgestellt werden, dass es einen Zusammenhang von Versicherungsbetrug und der Bindung an ein Unternehmen gibt (Meschkat und Nauert 2008). Durch eine längere Zusammenarbeit ist es möglich, dass sich die dem Menschen inhärente Verhaltensweise der Reziprozität entfalten kann. Je länger und damit auch je öfter Menschen die Möglichkeit einer aktiven Zusammenarbeit pflegen können, umso besser können sich ein belastbares Vertrauensverhältnis und direkte Reziprozität entwickeln.

\subsubsection{Prämienpolitik}

Ein weiteres Instrument der Absatzpolitik ist die Prämienpolitik. Die Prämienpolitik ist das „Bestimmen der Preise für die abzusetzenden bzw. abgesetzten Versicherungsprodukte" (Farny 2006, S. 679) und ein Ansatzpunkt zur Bekämpfung des moral hazards. Sowohl die ökonomische Theorie als auch die Ergebnisse der ultimaten Ver- 
haltensanalyse postulieren die Minimierung des Interessenkonflikts zwischen dem Versicherungsnehmer und dem Versicherungsunternehmen, um so das moralische Risiko zu verkleinern. Eine solche Minimierung kann im Zuge der Prämienpolitik stattfinden.

Ein Indiz für ausnützerisches Verhalten können überdurchschnittliche häufige und hohe Schadenzahlungen darstellen. Der Versicherungsnehmer kann nun für die hohen Schadenzahlungen mit der Erhöhung der Prämie, insbesondere der Risikoprämie, bestraft werden. Für den Versicherungsnehmer bedeutet dieses mögliche Resultat seines Verhaltens die Veränderung der persönlichen Auszahlungsmatrix. Die Restriktionen würde dessen Verhalten kurz- und langfristig für ihn unrentabel machen. Durch eine effektive Prämienpolitik kann aber auch eine Belohnung des Versicherungsnehmers für kooperatives Verhalten stattfinden. Unterdurchschnittliche Schadenzahlungen über einen längeren Zeitraum können darauf schließen lassen, dass der Versicherungsnehmer seinen Informationsvorsprung gegenüber dem Versicherungsunternehmen nicht ausnutzt. Die Senkung seiner Prämie kann dieses Verhalten honorieren und für die Zukunft dafür sorgen, dass zwischen dem Versicherer und dem Versicherungsnehmer kein Zielkonflikt besteht. Beide Maßnahmen werden von den Versicherungsunternehmen bereits eingesetzt. Überdurchschnittlich hohe Schäden können mit Hochstufungen der Tarifklasse, beispielsweise in der Kfz-Haftpflichtversicherung, bestraft und unterdurchschnittliche Schäden mit Schadenfreiheitsrabatten (Fürstenwerth und Weiß 2001) belohnt werden.

Diese Maßnahmen haben aber nur eine geringe Wirkung auf die bestehende Problematik (Nell 1993). Nach ökonomischer Begründung wird erwartet, dass formal institutionalisierte finanzielle Anreize einen positiven Einfluss auf das Verhalten des Einzelnen haben. Analog verhält es sich mit Bestrafungsmechanismen, welche zur Reduzierung eines bestimmten Verhaltens führen sollen. Verhaltensökonomische Untersuchungen präsentieren hierzu ein differenziertes Bild. Grundlage ist dabei die Hypothese, wonach sich der Mensch zu einem reziproken Wesen entwickelt hat und de facto der homo reciprocans an die Stelle des homo oeconomicus tritt (Göbel und Ortmann 2007). Ausgangspunkt der verhaltensökonomischen Überlegungen ist der dem Menschen inhärente starke Gerechtigkeitssinn. Dieser sorgt zu einer kritischen Hinterfragung der institutionellen Belohnungs- und Bestrafungsstrategien (West et al. 2007b). Anders als in der Ökonomie postuliert, können sich deswegen institutionalisierte finanzielle Anreize und Sanktionen aus verhaltensorientierter Sicht kontraproduktiv auf die Kooperationsbereitschaft des Menschen auswirken (Gneezy und Rustichini 2000).

Aufgrund der dem Menschen inhärenten reziproken Verhaltensweisen sollte eine über die gesamte Vertragsdauer kontinuierliche Prämiendifferenzierung als Belohnungs- oder Bestrafungsinstrumentarium mit Bedacht eingesetzt werden. Finanzielle Strafen können zwar abschreckend wirken, aber auch negative Effekte haben. Diese negativen Effekte können sowohl bei geringen als auch bei hohen finanziellen Sanktionen auftreten. Es kann sich daher als erfolgreicher erweisen, durch eine großzügige Prämienpolitik im Vorfeld auf die Reziprozität des Menschen zu vertrauen und so dessen defektionäres Verhalten einzuschränken. Fehr et al. (1997) haben diese These mit dem Ergebnis untersucht, dass Großzügigkeit im Vorfeld zwar das Ausnutzen von Informationsasymmetrie nicht vollständig verhindern kann, es im 
Großteil der betrachteten Fälle aber nicht zu einem vollständigen Opportunismus seitens der Individuen des Kollektivs kommt, d. h. sie verhalten sich tendenziell so, wie es die evolutionäre Kooperationsforschung erwarten lässt. Fehr und Gächter (2000a) haben die Auswirkung von Strafe und Belohnung experimentell überprüft und dabei festgestellt, dass das Vertrauen auf Reziprozität ein wirkungsvolleres Instrument als Belohnung und Strafe darstellen kann. Strafen und finanzielle Anreize können zum Teil sogar reziproke Verhaltensweisen eliminieren. Diese Kenntnisse können aber nicht nur in der Prämienfestsetzung, sondern sollten in allen Ex-ante-Leistungen des Versicherungsunternehmens umgesetzt werden.

\subsubsection{Servicepolitik}

Bestandteile der Service- oder Kundendienstpolitik sind alle Leistungen des Versicherungsunternehmens, die nicht vertraglich vereinbarte Hauptleistungen des Versicherungsgeschäfts sind, sondern zusätzlich gewährt werden (Farny 2006). Die Servicepolitik ist integraler Bestandteil der Absatzpolitik und kann in einem gewissen Maße als Teil des Absatzverfahrens angesehen werden. Servicemaßnahmen können nach dem Durchführungszeitpunkt klassifiziert werden. Es wird Service vor und bei Vertragsabschluss, Service während der Vertragsdauer und Service im Versicherungsfall unterschieden. $\mathrm{Zu}$ jedem Zeitpunkt stehen dem Versicherungsunternehmen Maßnahmen zur Verfügung, die eine kundenorientierte Absatzpolitik ermöglichen können.

Die Servicepolitik kann vor dem Hintergrund der Erkenntnisse der ultimaten Verhaltensanalyse ein wirkungsvolles Instrumentarium zur Minderung ausnützerischer Verhaltensweisen auf Kundenseite darstellen. Eine schwerpunktmäßige Servicepolitik mit Maßnahmen vor Vertragsabschluss und während der Vertragsdauer kann vom Versicherungsnehmer als reziproke Vorleistung interpretiert werden und so Auswirkung auf den Eintritt des Versicherungsfalles und dessen Höhe haben. Ein guter Service vor Schadeneintritt kann den Versicherungsnehmer dahingehend beeinflussen, auf eine aktive Schadenverhütung zu achten oder auf betrügerische Handlungen zu verzichten.

Wie erläutert, konnte Reziprozität evolvieren, da Individuen vorzugsweise mit jenen Subjekten agieren, die in der Vergangenheit vollständig reziproke Verhaltensweisen gezeigt haben (Röhl et al. 2007). Vor diesem Hintergrund kann ein guter Service im Versicherungsfall dem Versicherungsunternehmen zur Bildung einer positiven Reputation verhelfen. Maßnahmen wie Schadenschnelldienste oder Hilfsdienste zum Schutz versicherter Sachen können vom versicherten Individuum als Gegenleistung für seine Prämienzahlung interpretiert werden, die über die reine Schadenleistung hinausgeht. Diese Leistungen können nun zu einer Reputationssteigerung des Versicherungsunternehmens und zu einer in der Zukunft kooperativeren Verhaltensweise des Versicherungsnehmers führen. Wenn dem Individuum Reziprozität unterstellt wird, so wird es die Leistungen des Versicherers mit Verzicht auf Ausnutzung des Informationsvorsprungs zurückzahlen. Ein Umstand, der zu einem gewissen Maße auch experimentell belegt ist (Fetchenhauer 1998) und besonders im Zusammenhang mit dem Service nach dem Schadenfall auftritt.

Wichtig bei den Maßnahmen der Absatzpolitik im Allgemeinen und der Servicepolitik im Speziellen ist es, dem Versicherungsnehmer eine Diskriminierung des Ver- 
sicherers zu ermöglichen. Was bei dem Versicherungsunternehmen durch das Zurückgreifen auf technologische und organisatorische Elemente wie den Einsatz von Datawarehouses oder Uniwagnis erreicht wird, geschieht bei dem Versicherungsnehmer durch seine kognitiven Fähigkeiten. Die Wiedererkennung des Gegenübers und die Erinnerung an dessen Transaktionsgeschichte stellen die Voraussetzung für die Etablierung kooperativen Verhaltens und den Aufbau von Reputation dar. Im Zusammenhang mit dem sozialen Gehirn des Menschen scheint es daher wichtig, dass der Interaktionspartner ,Versicherung“ ein menschliches Gesicht bekommt. Die Diskriminierung auf Kundenseite kann dadurch erreicht werden, dass jedem Versicherten ein zentraler Ansprechpartner zugeordnet wird und dieser auch über die verschiedenen Prozesse erhalten bleibt. Experimente von Abric (1982) deuten darauf hin, dass eine subjektive Repräsentation einer Institution positiven Einfluss auf das kooperative Verhalten des interagierenden Kollektivs besitzt. Die subjektive Wahrnehmung der potentiell betrogenen Institution beeinflusst die allgemeine Einstellung zu einem Betrug dieser Institution.

\subsubsection{Kommunikationspolitik}

Kommunikationspolitik bezeichnet ,die Gestaltung der Informationen, die das Versicherungsunternehmen an seine generelle Umwelt und an einzelne Umweltsegmente abgibt“" (Farny 2006, S. 699). Wichtiger Bestandteil der Kommunikationspolitik ist die Öffentlichkeitsarbeit. Sie hat die systematische Pflege der Beziehungen zur Umwelt des Versicherungsunternehmens zur Aufgabe. Durch sie soll Verständnis für dessen Ziele und Aktivitäten hervorgebracht und Vertrauen begründet werden.

Wie dargelegt, hat sich der Mensch im Laufe der Evolutionsgeschichte zu einer reziproken Spezies entwickelt. Das Prinzip Versicherung basiert auf genau dieser Verhaltensweise: Durch die Vorleistung einer Prämienzahlung erhält der Versicherungsnehmer den Versicherungsschutz. Das moralische Risiko kann nun dadurch entstehen, dass das reziprok veranlagte Individuum als Gegenleistung nicht den Versicherungsschutz, sondern nur die Schadenzahlung ansieht. Abhilfe kann hier eine konsequente Kommunikationspolitik schaffen. Indem das Prinzip Versicherung der Öffentlichkeit kommuniziert wird, kann Verständnis bei den Versicherten geschaffen und durch die daraus resultierende positive Reziprozität das moralische Risiko gemindert werden. Wie dargelegt, hat sich die Manipulation zu kooperativen Verhaltensweisen als effektiv gegen ausnützerisches Verhalten erwiesen. Die Wirkung, die Manipulation durch Öffentlichkeitsarbeit auf opportunistisches Verhalten aufgrund von Informationsasymmetrie besitzt, lässt sich gut an einer Initiative der Londoner Verkehrsbetriebe sehen. Eine aktive Kommunikationspolitik über die Risiken des Schwarzfahrens und dessen Bedeutung für die Allgemeinheit konnte die Anzahl der Delikte signifikant senken (Schweizer Rück 1993).

Ein ähnliches Vorgehen wäre nun für die Versicherungswirtschaft ratsam. Die Öffentlichkeitsarbeit müsste primär die Kommunikation des Prinzips Versicherung beinhalten. Im Firmenkundengeschäft kann dies sogar individuell mittels persönlicher Schulungen der Versicherungskunden passieren. Dem versicherten bzw. zu versichernden Individuum sollte erläutert werden, dass die Gegenleistung für die Zahlung einer Versicherungsprämie im Erhalt von Versicherungsschutz liegt. Gemäß der 
evolutionären Verhaltensforschung würden die Individuen aufgrund der ihnen inhärenten Reziprozität zu einem gewissen Maße auf betrügerisches Verhalten verzichten. Diese Verhaltensweise kann noch verstärkt werden, indem den Individuen die individuellen und die gemeinschaftlichen Vorteile der Reziprozität nahegebracht werden. Betrügerisches Verhalten wird bei der Berechnung der Prämie nicht einkalkuliert und führt so zu einer direkten individuellen Belastung für den Versicherungsnehmer. Gleichzeitig untergräbt es den Grundgedanken der Solidarität, welches dem Prinzip Versicherung inhärent ist. Öffentlichkeitsarbeit, die die Gemeinhilfe als Ziel der Versicherung kommuniziert, sollte so aufgrund der Prosozialität und des Gerechtigkeitssinnes des Menschen (Gintis 2000) zu einer Reduktion defektionärer Verhaltensweisen führen.

Die Bedeutsamkeit von Öffentlichkeitsarbeit und Kommunikationspolitik kann anhand verhaltensökonomischer Experimente dargelegt werden. Einige Studien lassen vermuten, dass Entscheidungen von Personen stark von der Art der Präsentation abhängig sind. Dieser Zusammenhang ist auch in der Versicherungswirtschaft existent (Schoemaker und Kunreuther 1979). Eine durchdachte Kommunikationspolitik kann diesen Effekt ausnutzen und das Verhalten des Versicherungsnehmers zu einem gewissen Maße steuern. Kurz- und mittelfristige Maßnahmen scheinen dabei allerdings unfruchtbar zu sein. Es ist davon auszugehen, dass Wahrnehmungsverzerrungen verhältnismässig stabil sind (Theil 2002). Eine aktive Kommunikationspolitik, die die im Menschen evolvierten Verhaltensweisen in besonderem Maße berücksichtigt, sollte daher einen dauerhaften Bestandteil der Absatzpolitik darstellen.

\section{Ausblick}

Durch sich ständig verändernde Rahmenbedingungen und den zunehmenden Marktund Wettbewerbsdruck sind die Versicherer gezwungen, die Kosten- und Preisstruktur zu optimieren. Das moralische Risiko stellt für beides eine direkte Gefahr dar. Moral hazard führt zu einer Erhöhung der Schadenzahlungen, des größten Kostenblocks der Versicherungsunternehmen, und indirekt zu einem Anstieg der Risikoprämie und somit zur Verteuerung der Versicherungsprodukte. Aufgrund des erhöhten Preis- und Kostendrucks werden Maßnahmen zur effektiven Bekämpfung des moralischen Risikos in der Versicherungswirtschaft an Gewicht gewinnen. Es ist davon auszugehen, dass in den nächsten Jahren eine Entwicklung dahingehend stattfinden wird, bestehende Ansätze weiter zu verbessern und neue Theorien zur Handhabung der Problematik zu etablieren. Tiefgehende verhaltensorientierte Ansätze, die nicht nur beobachtetes Verhalten und Wirkursachen, also das Wie menschlichen Handelns berücksichtigen, sondern die auch dessen ursprüngliche Zweckursache, das Warum bestimmter Verhaltensweisen, analysieren, dürften dabei eine immer stärkere Beachtung finden und vermutlich in den Mittelpunkt dieser Maßnahmen rücken.

Open Access Dieser Artikel wird zu den Bedingungen der „Creative Commons Attribution Noncommercial License“ zur Verfügung gestellt. Damit ist eine nichtkommerzielle Nutzung, Verbreitung und Vervielfältigung erlaubt, sofern die Autoren des Artikels und die genaue Quelle angegeben sind. 


\section{Literatur}

Abric, J.-C.: Cognitive processes underlying cooperation: The theory of social representation. In: Derlega, V.J., Grzelak, J. (Hrsg.): Cooperation and helping behavior, S. 73-94., New York (1982)

Alexander, R.D.: The biology of moral systems: Foundations of human behavior. New York (1987)

Alpar, P., Niedereichholz, J. (Hrsg.): Data mining im praktischen Einsatz: Verfahren und Anwendungsfälle für Marketing, Vertrieb, Controlling und Kundenunterstützung. Braunschweig (2000)

Axelrod, R.: Die Evolution der Kooperation, Studienausg, 6. Aufl. Oldenburg (2005)

Bahlinger, T.: Wertsteigerung durch den Einsatz von Business Intelligence im Kunden- bzw. Risikomanagement von Banken. In: Fischer, M. (Hrsg.): Handbuch Wertmanagement in Banken und Versicherungen, S. 555-568. Wiesbaden (2004)

Barash, D.P.: Soziobiologie und Verhalten. Hamburg (1980)

Boesch, C.: Cooperative hunting in wild chimpanzees. Anim. Behav. 48, 653-667 (1994)

Boesch, C., Boesch, H., Vigilant, L.: Cooperative hunting in chimpanzees: Kinship or mutualism? In: Kappeler, P.M., van Schaik, C.P. (Hrsg.): Cooperation in Primates and Humans, S. 139-150. Heidelberg (2006)

Bühner, R. (Hrsg.): Management-Lexikon. Oldenburg (2001)

Burkart, J.M., Fehr, E., Efferson, C., van Schaik, C.P.: Other regarding preferences in a non-human primate: Common marmosets provision food altruistically. Proc. Natl. Acad. Sci. USA 104, 19762-19766 (2007)

Camerer, C.F., Loewenstein, G., Rabin, M. (Hrsg.): Advancesin Behavioral Economics. New York (2004)

Darwin, C.: On the Origin of Species. London (1859)

Dufwenberg, M.: An experimental test of direct and indirect reciprocity incase of complete and incomplete information. Berlin (2000)

Dugatkin, L.A.: Animal cooperation among unrelated individuals. Naturwissenschaften 89, 533-541 (2002)

Dunbar, R.I.M.: The Social Brain Hypothesis. Evol. Anthrop. 6(5), 178-190 (1998)

Dunbar, R.I.M., Knight, C., Power, C.: The Evolution of culture: An interdisciplinary view. Edinburgh (1999)

Erlei, M., Leschke, M., Sauerland, D.: Neue Instituionenökonomik, 2. Aufl. Stuttgart (2007)

Farny, D.: Versicherungsbetriebslehre, 4. Aufl. Karlsruhe (2006)

Fehr, E., Fischbacher, U.: Third party punishment and social norms. Evol. Hum. Behav. 25, 63-87 (2004)

Fehr, E., Fischbacher, U., Gächter, S.: Strong reciprocity, human cooperation, and the enforcement of social norms. Hum. Nature-Int. Bios. 13, 1-25 (2002)

Fehr, E., Gächter, S.: Cooperation and punishments in public good experiments. Am. Econ. Rev. 90, 980-94 (2000a)

Fehr, E., Gächter, S.: Fairness and retaliation: The economics of reciprocity. J. Econ. Perspect. 14(3), 159-181 (2000b)

Fehr, E., Gächter, S., Kirchsteiger, G.: Reciprocity as a contract enforcement device: Experimental evidence. Econometrica 65(4), 833-860 (1997)

Fehr, E., Rockenbach, B.: Detrimental effects of sanctions on human altruism. Nature 422, 137-140 (2003)

Fetchenhauer, D.: Versicherungsbetrug: eine theoretische und empirische Analyse betrügerischen Verhaltens gegenüber einem anonymen Geschädigten. Baden-Baden (1998)

Fischer, E.A.: The relationship between mating system and simultaneous hermaphroditism in the coral reef fish, Hypoplectrus nigricans. Anim. Behav. 28, 620-633 (1980)

Fischer, M. (Hrsg.): Handbuch Wertmanagement in Banken und Versicherungen. Wiesbaden (2004)

Frey, B.S., Benz, M.: Ökonomie und Psychologie: eine Übersicht. In: Frey, D., von Rosenstiel, L. (Hrsg.): Enzyklopädie der Wirtschaftspsychologie, S. 1-32. Göttingen (2001)

von Fürstenwerth, F., Weiß, A.: Versicherungs-Alphabet: (VA); Begriffserläuterungen der Versicherung aus Theorie und Praxis, 10. Aufl. Karlsruhe (2001)

Geissmann, T.: Vergleichende Primatologie. Springer, Heidelberg (2003)

Gesamtverband der Deutschen Versicherungswirtschaft e.V. (GDV) (Hrsg.): Statistisches Taschenbuch der Versicherungswirtschaft. Karlsruhe (2008a) 
Gesamtverband der Deutschen Versicherungswirtschaft e.V. (GDV) (Hrsg.): Hinweis- und Informationssystem der Versicherungswirtschaft, URL: http://www.gdv.de/Downloads/Themen/Das_Hinweis_und_Informationssystem_der_Versicherungswirtschaft.pdf, (Abruf: 15.11.2008) (2008b)

Geschwind, N.: Specializations of the Human Brain. Sci. Am. 241(3), 180-199 (1979)

Gintis H.: Strong reciprocity and human sociality. J. Theoret. Biol. 206, 169-179 (2000)

Gneezy, U., Rustichini, A.: A fine is a price. J. Legal Stud. 29(1), 1-18 (2000)

Göbel, E.: Neue Institutionenökonomik, Konzeption und betriebswirtschaftliche Anwendungen. Stuttgart (2002)

Göbel, M., Ortmann, G., Weber, C.: Reziprozität - Kooperation zwischen Nutzen und Pflicht. Managementforschung 17, 161-206 (2007)

Grossmann, M.: Über das subjektive Risiko. In: Farny, D. (Hrsg.): Wirtschaft und Recht der Versicherung, S. 81-97. Karlsruhe (1969)

Henke, W.: Stammesgeschichte des Menschen: eine Einführung. Berlin (1999)

Hill, R.A., Dunbar R.I.M.: Social network size in humans. Hum. Nat. 14(1), 53-72 (2003)

Immelmann, K. (Hrsg.): Psychobiologie: Grundlagen des Verhaltens. Stuttgart (1988)

Kappeler, P.M.: Verhaltensbiologie. Berlin (2006)

Kappeler, P.M., Pereira, M.E.: Primate life histories and socioecology. The University of Chicago Press, Chicago (2003)

Kirchgässner, G.: Homo Oeconomicus. Tübingen (1991)

Kotrschal, K.: Im Egoismus vereint?: Tiere und Menschentiere - das neue Weltbild der Verhaltensforschung. München (1995)

Krebs, D.L.: Evolution of moral dispositions in the human species. In: LeCroy, D., Moller, P. (Hrsg.): Evolutionary perspectives on human reproductive behavior. Ann. NY Acad. Sci. 907, 132-148. New York (2000)

Krebs, J.R.: Einführung in die Verhaltensökologie, 3. Aufl. Berlin (1996)

Krol, G.-J.: Ökonomische Verhaltenstheorie. In: May, H. (Hrsg.): Handbuch zur ökonomischen Bildung, S. 15-29. München (1992)

Langergraber, K.E., Mitani, J.C., Vigilant, L.: The limited impact of kinship on cooperation in wild chimpanzees. Proc. Natl. Acad. Sci. USA 104, 7786-7790 (2007)

Leimar, O., Hammerstein, P.: Evolution of cooperation through indirect reciprocity. Proc. Biol. Sci., R. Soc. Lond. Ser. B 268, 745-753 (2001)

Lewontin, R., Rose, S., Kamin, L.J.: Not in our genes: Biology, ideology and human nature. Harmondsworth, Middlesex (1984)

Mahr, W.: Ethik und Moral im Versicherungswesen. München (1972)

McFarland, D.: Biologie des Verhaltens. Weinheim (1989)

Meschkat, N., Nauert, R. (Hrsg.): Betrug in der Kraftfahrzeugversicherung. Köln (2008)

Milinski, M., Wedekind, C.: Working memory constrains human cooperation in the Prisoner's dilemma. Proc. Natl. Acad. Sci. USA 95, 13755-13758 (1998)

Nell, M.: Versicherungsinduzierte Verhaltensänderungen von Versicherungsnehmern: eine Analyse der Substitutions-, Moral Hazard- und Markteffekte unter besonderer Berücksichtigung der Krankenversicherung. Karlsruhe (1993)

Nell, M.: Das moralische Risiko und seine Erscheinungsformen. Frankfurter Vorträge zum Versicherungswesen 29, 7-33 (1998)

Nowak, M.A., Sigmund, K.: Evolution of indirect reciprocity by image scoring. Nature 393, 573-577 (1998)

Österreichische Finanzmarktaufsicht (FMA) (Hrsg.): Betriebsergebnisse „Feuer-Zivil“ (SchadenUnfallversicherung), URL: http://fma-va.brz.gv.at/pvaww/va.Tabelle?p_tabnr=34\&_ugl=1 \&p_zweigname=Feuer-Zivil\&p_rj=2007, (Abruf: 20.11.2008) (2008a)

Österreichische Finanzmarktaufsicht (FMA) (Hrsg.): Statistik der kleinen Versicherungsvereine 2007, URL: http://www.fma.gv.at/cms/site//attachments/9/6/4/CH0216/CMS1225716220063/ kurzstatistik_2007.xls, (Abruf: 29.11.2008) (2008b)

Packer, C.: Reciprocal altruism in Papio anubis. Nature 265, 441-443 (1977)

Panchanathan, K., Boyd, R.: A tale of two defectors: The importance of standing for evolution of indirect reciprocity. J. Theor. Biol. 224, 115-126 (2003)

Parsons, C.: Moral Hazard in liability insurance. Geneva Pap. Risk Insur. 28(3), 448-471 (2003) 
Pease, A., Pease, B.: Warum Männer nicht zuhören und Frauen schlecht einparken: Ganz natürliche Erklärungen für eigentlich unerklärliche Schwächen. Ullstein (2000)

Pirk, K., Wolf, V.: Business Intelligence von IBM. In: Schütte, R. et al.: Data-warehouseManagementhandbuch: Konzepte, Software, Erfahrungen, S. 207-232. Berlin (2001)

Rabin, M.: A perspective on psychology and economics. Berkeley (2002)

Röhl, T., Röhl, C., Schuster, H.G., Traulsen, A.: Impact of fraud on the mean-field dynamics of cooperative social systems. Phys. Rev. E 76(3), 030101 (2007)

Schino, G.: Grooming and agonistic support - a meta-analysis of primate reciprocal altruism. Behav. Ecol. 18, 115-120 (2007)

Schoemaker, P.J.H., Kunreuther, H.C.: An experimental study of insurance decisions. J. Risk Insur. 46, 603-618 (1979)

Schütte, R., Rotthowe, T., Holten, R. (Hrsg.): Data-warehouse-Managementhandbuch: Konzepte, Software, Erfahrungen. Berlin (2001)

Schwarze, R.: Risikopolitische Instrumente zur Kontrolle des moralischen Risikos bei verdeckten Langzeitschäden am Beispiel der Umwelthaftpflichtversicherung. In: Männer, L. (Hrsg.): Langfristige Versicherungsverhältnisse, S. 331-355. Karlsruhe (1997)

Schweizer Rück (Hrsg.): Massendelikt Versicherungsbetrug. Zürich (1993)

Semmann, D., Krambeck, H.-J., Milinski, M.: Volunteering leads to rock-paper-scissors dynamics in a public goods game. Nature 425, 390-393 (2003)

Semmann, D., Krambeck, H.-J., Milinski, M.: Reputation is valuable within and outside one's own social group. Behav. Ecol. Sociobiol. 57, 611-616 (2005)

Silk, J.B.: Practicing Hamilton's rule: Kin selection in primate groups. In: Kappeler, P.M., van Schaik, C.P. (Hrsg.): Cooperation in Primates and Humans, S. 25-46. Heidelberg (2006)

Silk, J.B.: Social components of fitness in primate groups. Science 317, 1347-1351 (2007)

Söllner, F.: Die Geschichte des ökonomischen Denkens, 2. Aufl. Berlin (2001)

Sommer, V.: Lob der Lüge. Täuschung und Selbstbetrug bei Tier und Mensch. München (1992)

Stegbauer, C.: Reziprozität: Einführung in soziale Formen der Gegenseitigkeit. Wiesbaden (2002)

Streitferdt, L.: Grundlagen und Probleme der betriebswirtschaftlichen Risikotheorie. Wiesbaden (1973)

Theil, M.: Versicherungsentscheidungen und prospect theory: Die Risikoeinschätzung der Versicherungsnehmer als Entscheidungsgrundlage. Wien (2002)

Tinbergen, N.: Instinktlehre: vergleichende Erforschung angeborenen Verhaltens. Berlin, Hamburg (1979)

Trivers, R.: The evolution of reciprocal altruism. Q. Rev. Biol. 46, 35-57 (1971)

Tucker, T., Ferson, S.: Evolved altruism, strong reciprocity and perception of risk. Ann. NY Acad. Sci. 1128, 111-120 (2008)

Voland, E.: Grundriss der Soziobiologie, 2. Aufl. Heidelberg, Berlin (2000)

Wagner, F., Warmuth, W.: Wertorientierte Bepreisung im Versicherungsgeschäft. Karlsruhe (2005)

Warneken, F., Hare, B., Melis, A.P., Hanus, D., Tomasello, M.: Spontaneous altruism by chimpanzees and young children. PLOS Biology 5, 1414-1420 (2007)

West, S.A., Griffin, A.S., Gardner, A.: Social semantics: Altruism, cooperation, mutualism, strong reciprocity and group selection. J. Evol. Biol. 20(2), 415-432 (2007a)

West, S.A., Griffin, A.S., Gardner, A.: Evolutionary explanations for cooperation. Curr. Biol. 17, R661-R672 (2007b)

Winkler, E.-M.: We are just modelling. Über die Bedeutung der Soziobiologie für die Anthropologie. In: Ehalt, H.C. (Hrsg.): Zwischen Natur und Kultur, S. 43-61. Böhlau, Wien (1985)

Wuketits, F.M.: Soziobiologie. Die Macht der Gene und die Evolution sozialen Verhaltens. Heidelberg (1997)

von Zedtwitz, C.: Die rechtsgeschichtliche Entwicklung der Versicherung. Zürich (1999)

Zweifel, P., Eisen, R.: Versicherungsökonomie, 2. Aufl. Berlin (2003) 